\title{
Monitoring antiangiogenesis of bevacizumab in zebrafish
}

This article was published in the following Dove Press journal:

Drug Design, Development and Therapy

\author{
Jing Zhang ${ }^{1,2, *}$ \\ Beili Gao',* \\ Wenchao Zhang' \\ Zijun Qian' \\ Yi Xiang'
}

'Department of Respiratory and Critical Care Medicine, Ruijin

Hospital, Institute of Respiratory Diseases, School of Medicine, Shanghai Jiaotong University, Shanghai, People's Republic of China; ${ }^{2}$ Respiratory

Department of Shanghai East Hospital Affiliated to Tongji University, Shanghai, People's Republic of China

*These authors contributed equally to this work
Correspondence: Yi Xiang

Department of Respiratory and Critical Care Medicine, Ruijin Hospital, Institute of Respiratory Diseases, School of Medicine, Shanghai Jiaotong University, No 197 Ruijin 2nd Road, Shanghai, People's Republic of China Email xiangyiht@I63.com

\begin{abstract}
Bevacizumab, which is a humanized anti-VEGF antibody, has been successfully applied in clinics since 2004. Bevacizumab in combination with chemotherapy showed high safety and has been applied to solid tumors. However, studies on the insight into the mechanism about the antiangiogenesis activity of bevacizumab were mostly done on mice models, and so there are no visual and intuitive models to observe the process of antiangiogenesis. Here, we first used a zebrafish model to investigate the angiogenesis suppressing behavior of bevacizumab. Our results showed that bevacizumab inhibited formation of zebrafish subintestinal veins, which mimics the process of tumor angiogenesis in vivo. Meanwhile, bevacizumab caused specific vasculature formation defects in subintestinal veins but not in the trunk. Our study also indicated that bevacizumab could inhibit zebrafish retinal angiogenesis with therapeutic potential.
\end{abstract}

Keywords: zebrafish, bevacizumab, anti-angiogenesis, VEGF, subintestinal vein

\section{Introduction}

In 1971, it was hypothesized by Folkman that tumor cells released signals to their adjacent vascular endothelial cells (ECs) which then promote neovascularization. Without neovascularization, the growth of most solid tumors will cease at 2-3 mm in diameter and then enter into a dormant state. ${ }^{1}$ Detaching from the primary site to other organs is the prerequisite for tumor cell metastasis, which is what is offered by the tumor neovascularization. ${ }^{2}$ As angiogenesis is critical for tumor growth and metastasis, new drugs based on inhibiting angiogenesis are very promising in clinics. Currently, dozens of antiangiogenesis drugs are being applied in the clinic or verified in clinical trials. ${ }^{3}$

The process of angiogenesis is regulated by pro- and antiangiogenic factors, in which various proteins are highly coordinated. Once the balance is broken, neovascularization occurs, which promotes tumor growth and metastasis. Among the numerous cytokines attribute to angiogenesis, vascular endothelial growth factor (VEGF) is key. VEGF exerts biological functions through binding with its tyrosine kinase receptors, including VEGFR-1, VEGFR-2, and VEGFR-3. After VEGF binds to its receptors, ECs can be activated and produce matrix metalloproteinases to disrupt the extracellular matrix, which allows ECs to proliferate and transdifferentiate, resulting in neovascularization. ${ }^{4}$ Bevacizumab is a humanized monoclonal antibody which prevents VEGF from interacting with VEGFR-1 and 2 and eventually inhibits ECs proliferation and angiogenesis. ${ }^{5}$ It is licensed to treat solid tumors, including colorectal, lung, and breast cancer and glioblastoma. ${ }^{6-8}$ Bevacizumab, as an angiogenesis inhibitor, was first used in clinics in the USA. It was first approved in 2004 to be used with standard chemotherapy for the treatment of metastatic colon cancer. ${ }^{9}$ This pioneer of antiangiogenesis therapy had seen great success in metastatic colorectal 
cancer, which until now responds well to bevacizumab. ${ }^{10}$ The compelling evidence for usage of bevacizumab with a fluoropyrimidine demonstrates its efficacy and safety, which continually prolongs survival for patients with this cancer. ${ }^{9}$ Bevacizumab has been approved by the China Food and Drug Administration for the treatment of metastatic colorectal cancer and non-small-cell lung cancer, in combination with chemotherapy. The success of bevacizumab seen in most Chinese people promoted the further exploration of its activity. However, previous mouse model studies cannot provide a visual process of antiangiogenesis induced by bevacizumab.

Currently, zebrafish has emerged as a valuable model organism to substitute traditional models to assay angiogenesis inhibitors. Exploration of the cardiovascular system in transparent zebrafish embryos and larvae provides a unique opportunity to directly observe blood flow and vascular development without resorting to sophisticated instrumentation. Genomics studies have shown that the signaling pathways in fish and mammals are conserved. Therefore, research focused on the biological characteristics of the vascular system in zebrafish can be directly converted to related information for human diseases. Since the generation of the first stable transgenic zebrafish line was reported, hundreds of transgenic lines have been developed. ${ }^{11}$ The transgenic zebrafish lines, Tg(fli1a:EGFP)y, ${ }^{1}$ make it possible to observe the antiangiogenesis activity visually. During embryogenesis, the expression of enhanced green fluorescent protein (EGFP) in vascular system is under the control of the zebrafish flil promoter.

The development of the vascular structure of zebrafish showed high similarity to other vertebrates, as demonstrated by previous evidence. ${ }^{12,13}$ The process of blood vessel formation in zebrafish embryo can be made to simulate angiogenesis seen in cancers and other benign lesions. Also, the intersegmental vessels (ISVs) of the trunk and subintestinal veins (SIVs) in the zebrafish embryos had usually been used as evaluation indicators for antiangiogenesis inhibitors. During embryogenesis, cells exit the dorsal aorta and migrate between the somite boundaries to initiate sprouting at about $20 \mathrm{~h}$ postfertilization (hpf). Once cells reach the horizontal myoseptum, the segmental artery tip cell undergoes a single cell division (20.5-23.5 hpf), after which one cell maintains its position and becomes a connector cell while the tip cell continues to migrate dorsally and forms the dorsal longitudinal anastomotic vessel (30-31 hpf). By 2 days postfertilization (dpf), majority of the trunk and tail ISVs have formed lumina and involved in blood circulation. ${ }^{14,15}$ At 2 dpf, SIVs are derived from the duct of Cuvier. Between 2 and $3 \mathrm{dpf}$, a vascular plexus from SIVs will be formed across most of the dorsal-lateral aspect of the yolk ball. ${ }^{16}$
Previous studies had verified that bevacizumab specifically bound human VEGF. However, it is still unknown whether it influences zebrafish VEGF. ${ }^{17,18}$ So we tried to develop a method to rapidly analyze antiangiogenesis monoclonal antibody in a live fluorescent zebrafish and assay the efficacy of bevacizumab in the zebrafish model.

\section{Materials and methods Zebrafish care and maintenance}

All animal procedures were approved by the ethical committee of Institute of Respiratory Diseases, Shanghai Jiaotong University School of Medicine, Shanghai, People's Republic of China, and conducted in full compliance with the guidelines of the Animal Care Committee. Adult zebrafish were maintained at $28.5^{\circ} \mathrm{C}$ on a $14 \mathrm{~h}$ light $/ 10 \mathrm{~h}$ dark cycle. Five to 6 pairs of zebrafish were maintained at $28.5^{\circ} \mathrm{C}$ on a $14 \mathrm{~h}$ light $/ 10 \mathrm{~h}$ dark cycle for nature mating each time. About 200-300 embryos were produced on average and cultured at $28.5^{\circ} \mathrm{C}$ in fish water. The embryos were washed and staged as Kimmel described. ${ }^{19}$ A flila-EGFP transgenic line was used, according to a previous study. ${ }^{20}$ The zebrafish facility at Shanghai Research Center for Model Organisms was approved by the Association for Assessment and Accreditation of Laboratory Animal Care International.

\section{Drug administration}

Bevacizumab (Avastin, Roche, Basel, Switzerland) solution was injected into the yolk sac, from where protein is usually absorbed by the embryo, so that the protein eventually entered the embryonic circulation after injection. The experimental procedure was carried out as follows: 1 and $2 \mathrm{mg} / \mathrm{mL}$ solution of bevacizumab in PBS was back-filled into a pulledglass micropipette. The micropipette was then attached to a micromanipulator (World Precision Instruments, Sarasota, FL, USA; M3301R) and a microinjector (Eppendorf, Hamburg, Germany; FemtoJet). ${ }^{21}$ Approximately $40 \mathrm{~nL}$ of bevacizumab solution was injected into the yolk sac for each embryo. Injected embryos were transferred to a 6-well plate (BD Falcon, BD Biosciences, Franklin Lakes, NJ, USA) in $10 \mathrm{~mL}$ fresh fish water and kept at $28.5^{\circ} \mathrm{C}$ until $4 \mathrm{dpf}$ when the experiments were terminated, and animals were euthanized by overexposure to tricaine.

\section{Zebrafish angiogenic assessment}

To calculate blood vessels formation, $2.5 \mathrm{dpf}$ embryos were treated with bevacizumab for a treatment period of $36 \mathrm{~h}$, while matched groups were injected with the equal amount of PBS solution. All zebrafish were cultured at $28.5^{\circ} \mathrm{C}$. After treatment, embryos were anesthetized using MS-222 
(tricaine methanesulfonate) according to previous study. ${ }^{22}$ The area of the SIVs and the average number of blood vessel segments per eye were calculated using the NIS-Elements D3.1 software (Nikon SMZ1500 Fluorescence microscope, Tokyo, Japan). To evaluate the number of blood vessels per retina, we counted the number of vessel segments that met the following criteria: were characterized by roughly parallel walls, displayed distinct lumen, were open at both ends, and stretched for a minimum of 15 arbitrary length units (measured in pixels on Photoshop images). The results of these counts are provided in Figure 1. Clumps of GFPpositive tissue that did not have a lumen or were not open at both ends were considered "collapsed vessels" and were not counted.

\section{Image acquisition}

Referring to a previous study, Nikon SMZ 1500 Fluorescence microscope and a digital camera were used for analysis and to take photographs orderly. NIS-Elements D3.1 was used to quantify the image. Adobe Photoshop 7.0 software (Adobe, San Jose, CA, USA) was used to adjust the level, brightness, contrast, hue, and saturation of a series of images to optimize the visual representation. ${ }^{23}$

\section{Statistical analysis}

GraphPad Prism 5.0 (GraphPad Software, La Jolla, CA, USA)

was used to analyze data and draw graphics. All statistics were expressed as the mean \pm standard error of mean (SEM) and analyzed by unpaired Student's $t$-test or one-factor analysis of variance. Two-sided $P$-values $<0.05$ were considered statistically significant $(* P<0.05$, and $* * * P<0.0001)$.

\section{Results}

\section{Bevacizumab inhibited formation of zebrafish SIVs in a dose-dependent manner}

To determine if bevacizumab has any effect on zebrafish angiogenesis, we established and characterized the flila-EGFP transgenic lines as described previously. ${ }^{20}$ As mentioned above, the expression of EGFP could be driven by the fli1 promoter in all blood vessels in embryo and juvenile animals. EGFP expression is detected in both patent and forming blood vessels, as well as in migratory angioblasts that have not yet aggregated into vascular structures. In this respect, these transgenic lines allow confocal microangiography to visualize the development of blood vessels. ${ }^{20} \mathrm{We}$ chose ISVs of the trunk and SIVs in the zebrafish embryos to assay the efficacy of bevacizumab, as a previous study mentioned. ${ }^{24}$ We treated zebrafish embryos with bevacizumab at the pectoral fin stage ( $2.5 \mathrm{dpf}$ ). Figure 2 shows the representative fluorescent images of control or embryos treated with bevacizumab at $3 \mathrm{dpf}$. In control embryos, SIVs developed as a smooth basket-like structure over the yolk at $3 \mathrm{dpf}$. Blood vessels lined in an orderly manner and the integrity was well maintained (Figure 2A).
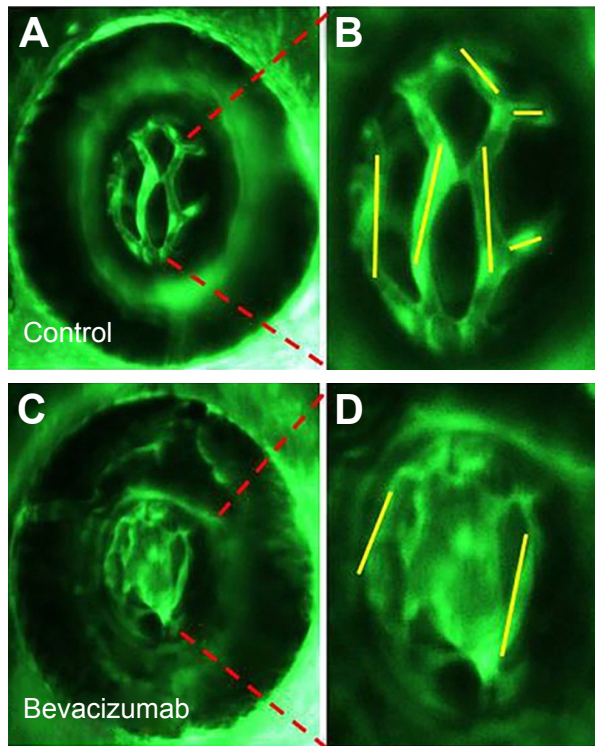

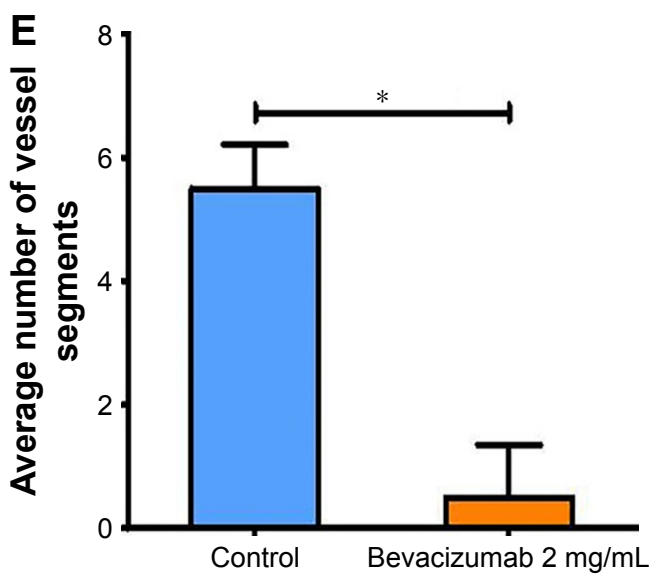

Figure I Bevacizumab inhibits zebrafish retinal angiogenesis.

Notes: (A-D) Representative fluorescent images of control or embryos treated with bevacizumab at 4 dpf. In the control image panel, yellow lines indicate the vessel segments. Compared with control, higher magnification of the retinae revealed that collapsed vessels can be observed after bevacizumab treatment. (E) Quantification of the average number of blood vessel segments per eye shows a II-fold decrease in bevacizumab-treated embryos $(n=10)$ at 4 dpf. *Refers to $P<0.000 \mathrm{I}$ by an unpaired Student's $t$-test.

Abbreviation: dpf, days post fertilization. 

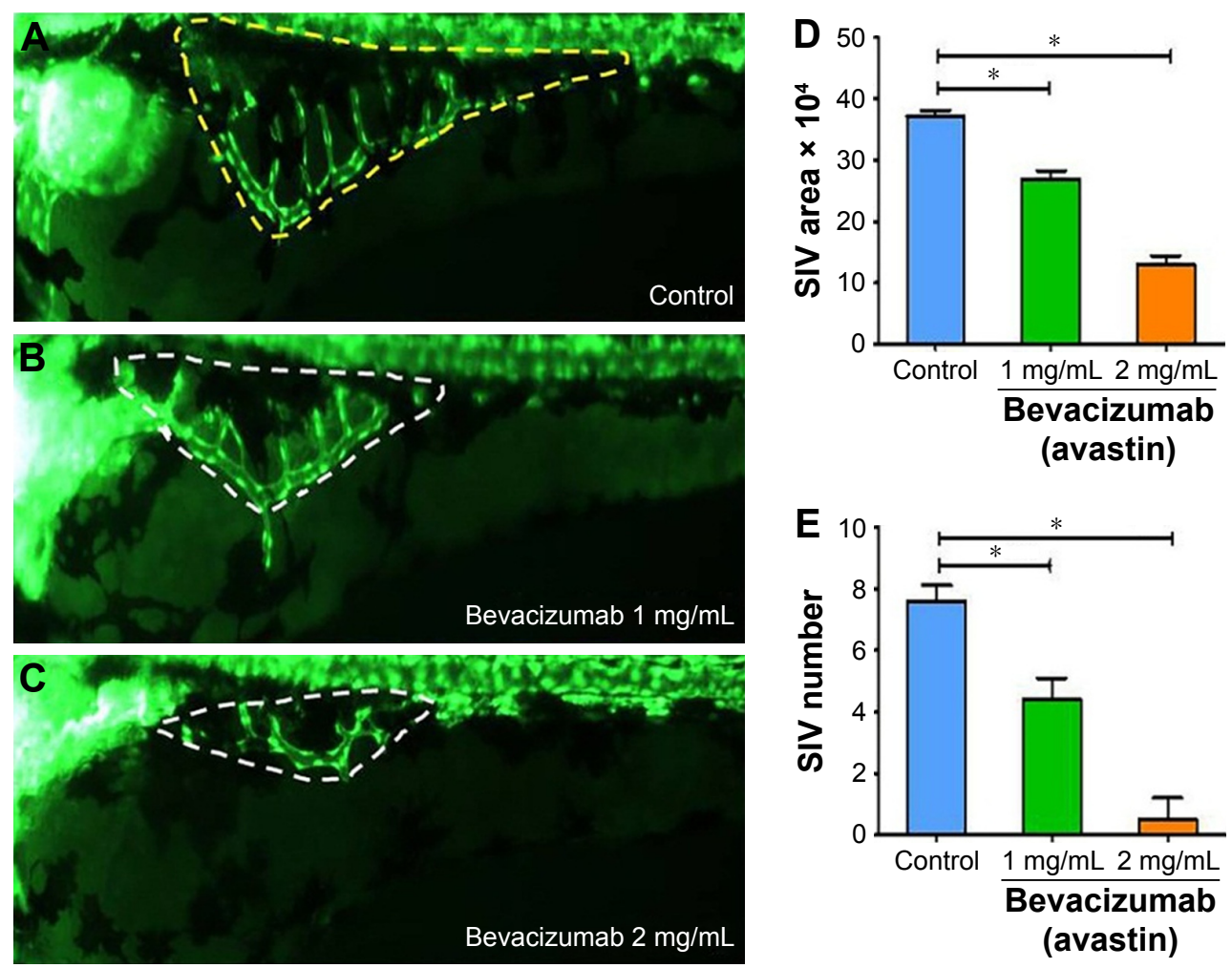

Figure 2 Bevacizumab impairs formation of zebrafish SIV in a dose-dependent manner.

Notes: Representative fluorescent images of control or embryos treated with bevacizumab at 3 dpf. In control embryos, SIVs developed as a smooth basket-like structure over the yolk at $3 \mathrm{dpf}$ (A, yellow dashed lines). In contrast, embryos treated with bevacizumab resulted in specific defects in SIVs formation (B, C, white dashed lines). (D, E) Quantification of the area and number of SIV show significant decrease in bevacizumab-treated embryos. Error bars, SEM; $* P<0.000$ I ( $n=10$; ANOVA).

Abbreviations: ANOVA, analysis of variance; dpf, days post-fertilization; SIV, subintestinal vein; SEM, standard error of mean.

In contrast, embryos treated with bevacizumab showed reduced vessel numbers and the structure was disordered (Figure 2B and C). Bevacizumab inhibited formation of zebrafish SIVs in a dose-dependent manner (Figure 2D and E).

\section{Bevacizumab caused specific vasculature formation defects in the SIVs}

We chose $2.5-3 \mathrm{dpf}$ as the treatment window of bevacizumab. As shown in Figure 3, when embryos were treated
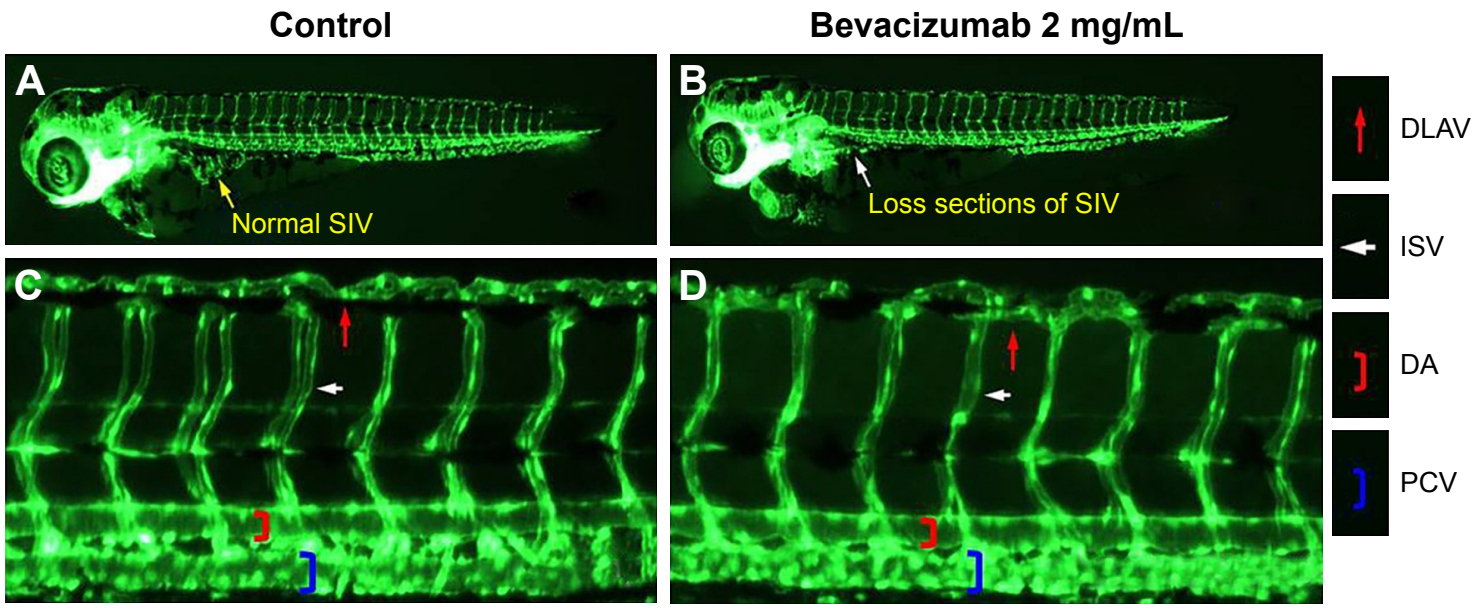

Figure 3 Bevacizumab causes specific vasculature formation defects in the SIV.

Notes: (A-D) Representative fluorescent images of zebrafish embryos at $3 \mathrm{dpf}$ treated with PBS (control) or bevacizumab (2 mg/mL). (A, B) In contrast, treatment with bevacizumab at the pectoral fin stage ( $2.5 \mathrm{dpf}$ ) causes specific vasculature formation defects in the SIV (B, white arrow). (C-D) Truncal vasculature of the zebrafish shows no appreciable phenotypic differences relative to control.

Abbreviations: DA, dorsal aorta; DLAV, dorsal longitudinal anastomotic vessels; ISV, intersegmental vessels; PCV, posterior cardinal vein; SIV, subintestinal vein; dpf, days post-fertilization. 
with bevacizumab in this window, specific vasculature formation defects in the SIVs were observed (Figure 3A and B), but there was no significant effect on the existed trunk vessels including ISVs, dorsal longitudinal anastomotic vessel, dorsal aorta, posterior cardinal vein (PCV) PCV (Figure 3C and D). However, the vascular defect in SIVs resulted in further complications, such as pericardial edema (Figure 4A-D).

\section{Bevacizumab inhibited zebrafish retinal angiogenesis}

As is well known, pathological angiogenesis in the retina is a key component of human irreversible blindness resulting from different eye diseases. Retinopathy of prematurity, diabetic retinopathy, and age-related macular degeneration are the contributing factors that lead to vision impairment or acquired blindness at different ages, including in children and adults. ${ }^{25-27}$ These common ocular disorders are characterized by overgrowth of disorganized, leaky, and physiologically nonfunctional retinal vessels, which result in serious vision impairment and blindness. The inner retina of zebrafish is nourished by an intricate vascular network which shares many features with human hyaloid and retinal vasculatures. ${ }^{27}$ It is worth mentioning that, unlike rodents, zebrafish has rich color vision, making it possible to mimic the macular degenerations. ${ }^{28}$ Thus, the zebrafish may provide an invaluable experimental model for investigating underlying mechanisms of retinal pathological angiogenesis, identifying potential therapeutic targets and drug evaluation. When treating zebrafish embryos at $2.5 \mathrm{dpf}, 36 \mathrm{~h}$ later, we chose the representative bright field and fluorescent images of zebrafish embryos treated with PBS (control) or bevacizumab ( $2 \mathrm{mg} / \mathrm{mL}$ ). The results showed that bevacizumab inhibited zebrafish retinal angiogenesis (Figure 5). The formation of vessels in control retinal embryos was normal and retinal vascular structures in the bevacizumab group were obscure. On quantitatively analyzing embryonic retinal blood vessels (Figure $1 \mathrm{~A}-\mathrm{E}$ ), we found that the Mean $\pm \mathrm{SEM}$ of retinal blood vessels in bevacizumab zebrafish embryos was $5.50 \pm 0.70$. However, the Mean \pm SEM of retinal blood vessels in control zebrafish embryos was $0.50 \pm 0.85, P<0.0001$, and there existed significant statistical difference.

\section{Discussion}

Angiogenesis has become a hot research topic in the field of targeted therapy due to its important role in different pathological settings, such as in solid tumors, age-related macular degeneration,
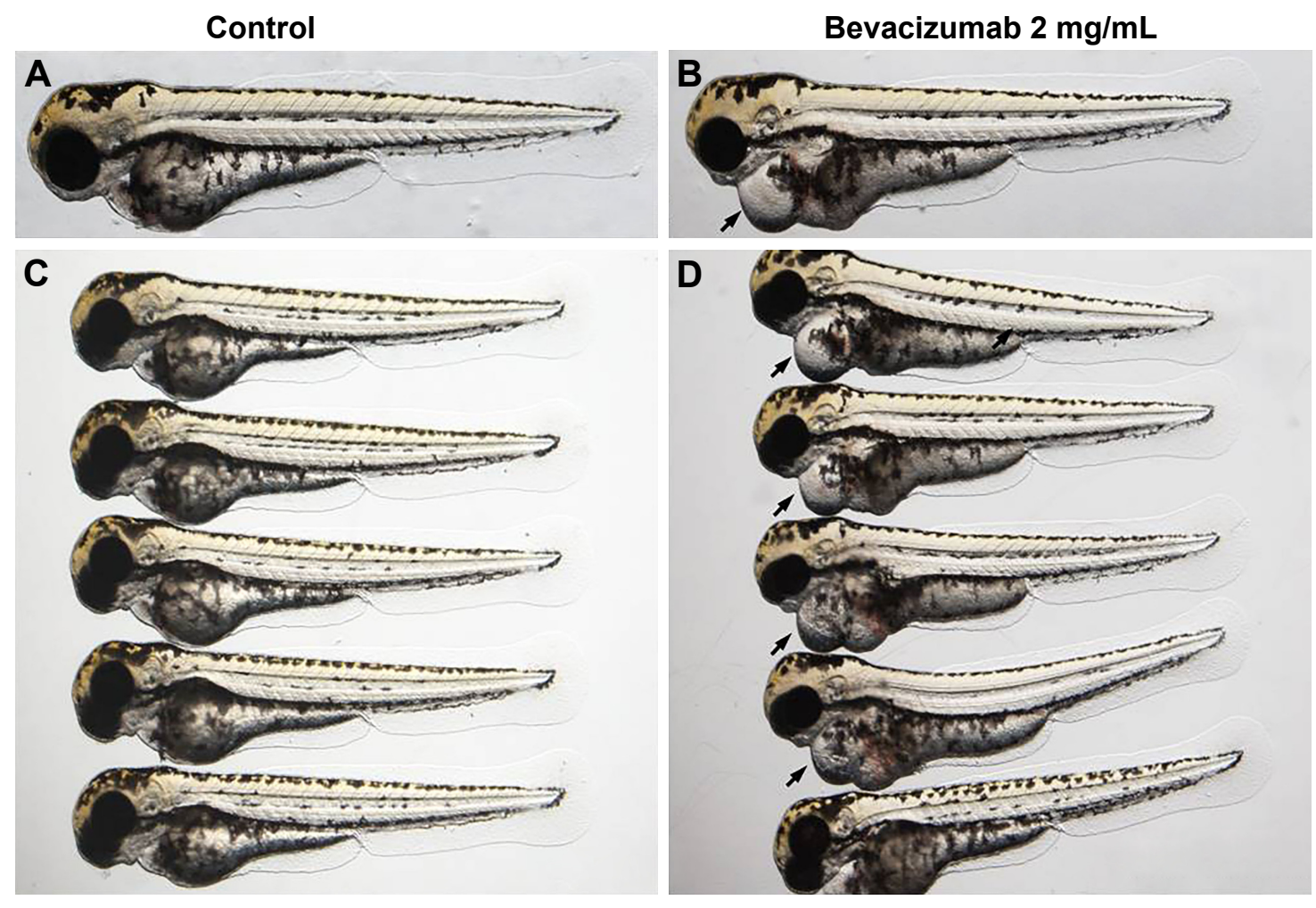

Figure 4 The vascular defect in SIV (subintestinal vein) resulted in further complications such as severe pericardial edema.

Notes: (A-D) Representative bright field images of zebrafish embroys at 3 days post-fertilization (dpf) treated with PBS (control) or bevacizuamb (2 mg/mL). The vascular defect in SIV resulted in further complications such as severe pericardial edema (B, black arrow) after bevacizumab treatment. Circulation in the SIV is visible in the control fish, but is absent in the bevacizumab-treated zebrafish. 

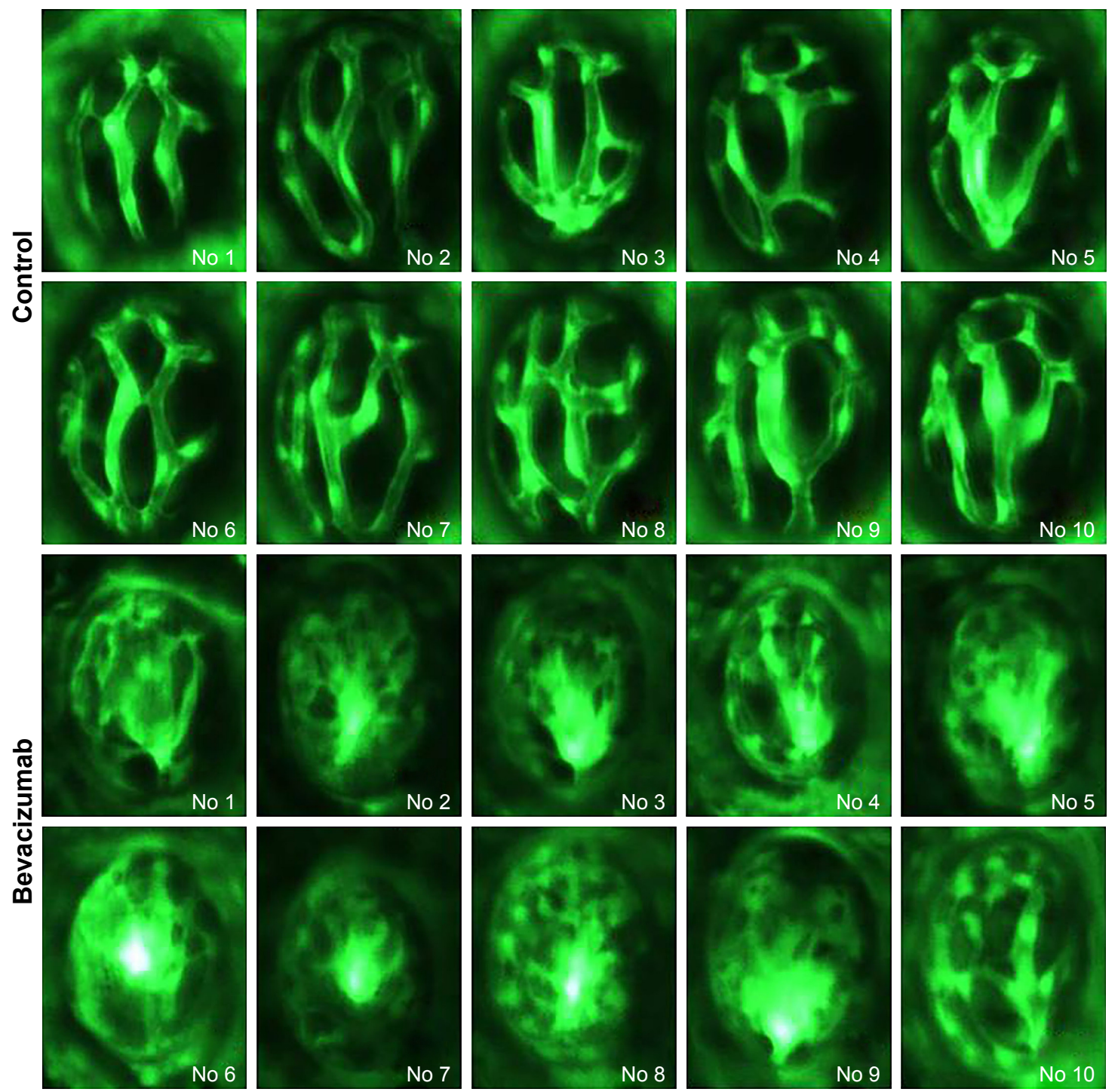

Figure 5 Representative fluorescent images of zebrafish retinal angiogenesis at $4 \mathrm{dpf}$ treated with control (PBS) and bevacizumab (2 mg/mL).

Note: Each row shows 5 examples of the phenotype induced in retina.

Abbreviation: dpf, days post fertilization.

rheumatoid arthritis, and endometriosis. ${ }^{29-32}$ Conventional angiogenic assays suitable for large-scale compound screening consist of in vitro cell-based models that lack the biological complexity associated with blood vessels growing in their natural environment. In contrast, traditional in vivo models - such as the chick chorioallantoic membrane assay and the corneal model in mice - are expensive, laborious, technically challenging, low throughput, and require comparatively large quantities of the test drug. ${ }^{33}$

Experimental evidences have indicated that developmental angiogenesis in the zebrafish embryo might provide a target for the screening of antiangiogenic compounds. The main merits of zebrafish for putative antiangiogenic drug discovery include their high genetic, physiologic, and pharmacologic similarity with humans. ${ }^{34}$ In addition, the small size, easy manipulation, rapid propagation, and large numbers of zebrafish embryos and larvae allow for a large-scale experiment. Due to the small size of the zebrafish embryos and larvae, experiments can be manipulated in microtiter plates for screening, in which only microgram amounts of drug for testing need to be added to the fish water. Due to the high fecundity of zebrafish, large numbers of embryos and larvae can be produced and analyzed in a more costeffective manner than other models such as mice and rats. As stated above, these advantages make zebrafish the ideal in vivo model for a rapid screening of novel antiangiogenesis inhibitors with therapeutic potential.

Transgenic technology has improved the inherent in vivo imaging capabilities that zebrafish embryos may provide for 
researchers. The availability of transgenic lines that express tissue-specific fluorescent proteins in vascular system make vessels and blood flow easily observable in great detail,${ }^{11}$ for example, the use of transgenic $\operatorname{Tg}\left(\right.$ flila:EGFP)y ${ }^{1}$ zebrafish embryos, in which ECs express the EGFP under the control of the fli1 promoter allowing the observation and time-lapse recording of newly formed blood vessels in live embryos by in vivo confocal microscopy.

However, when compared to other animal models for angiogenesis assessment, zebrafish also has some limitations such as the embryos are too small to extract and also the problem that several techniques cannot be applied including histochemistry and immunohistochemistry. At the early stage (48-72 hpf) of the zebrafish, the embryonic immune system is not mature enough to cause transplant rejection. However, during the development of zebrafish embryos, the progressively maturing immune system rejects the graft and the larvae eventually die, thus impeding the possibility of long-term tumor vascularization studies performed on this model. ${ }^{16}$

Bevacizumab is a humanized monoclonal antibody derived from A4.6.1, which primarily antagonizes human VEGF and has a very weak reactivity against murine VEGF. ${ }^{35}$ Our results revealed that bevacizumab significantly inhibited the formation of zebrafish SIVs, which in turn validated that zebrafish has highly similarity with humans in respect to VEGF, thus forming the basis for further study about molecular pathways related to angiogenesis. Unfortunately, bevacizumab has strong blocking activity against VEGFAinduced VEGFR2 protein phosphorylation. This blockade activity changed at protein phosphorylation levels, but not at mRNA expression levels. So, reverse transcription-PCR as a method is not suitable to detect the molecular mechanism of bevacizumab. But because of a lack of zebrafish-specific antibodies to detect protein phosphorylation, the use of Western blot is also hampered. Our team is now devoted to overcoming this obstacle; it may take some time, and we hope to present relevant results as soon as possible.

According to the existing evidences, several antiangiogenesis inhibitors represented by bevacizumab could reverse the disordered vascular structure of the tumor (rather than destroying vessels completely), thus normalizing the tumor microenvironment and improving sensitivity to radiotherapy or chemotherapy, eventually hampering tumor development and metastasis. However, the inhibition of zebrafish embryo angiogenesis by bevacizumab is irreversible, and this limits the experimental study related to tumor vascular normalization performed on zebrafish.
Above all, zebrafish embryo may represent a simple, cost-effective, and rapid model system for the discovery and validation of putative or classic compounds of antiangiogenic therapeutics and for the identification of genes associated with angiogenesis in different pathological diseases. But it may not completely replace all other traditional experimental models due to the abovementioned constraints.

\section{Conclusion}

In the current study, we used transgenic zebrafish to create a rapid, quantitative, phenotype-based angiogenesis assay capable of identifying the antiangiogenic activity of bevacizumab in a whole organism. To our knowledge, this was the first exploration that adapted zebrafish embryos to study the antiangiogenic effect of bevacizumab. As there was no reference on doses of macromolecular protein drugs to be used in the zebrafish, based on related literature, ${ }^{36}$ we chose 1 and $2 \mathrm{mg} / \mathrm{mL}$ bevacizumab to treat zebrafish embryos. The experimental results showed that bevacizumab inhibited the formation of zebrafish SIVs in a dose-dependent manner. Furthermore, when we treated embryos with bevacizumab at the pectoral fin stage ( $2.5 \mathrm{dpf})$, specific vasculature formation defects in the SIVs but not in the trunk were observed. In clinics, many doctors may worry that bevacizumab may affect vessels in normal tissue, but our results have revealed that bevacizumab had no effect on existing vessels, thus offering evidence of its safety.

Numerous studies have confirmed that VEGF plays an important role in pathological angiogenesis of the retina, and anti-VEGF agents have emerged for the treatment of retinal neovascularization. ${ }^{37,38}$ Bevacizumab is a classic angiogenesis inhibitor. Our study visually showed that bevacizumab could inhibit zebrafish retinal angiogenesis. The transgenic zebrafish lines provide great convenience for preclinical verification of antiangiogenic drugs, thus facilitating the treatment of pathological angiogenesis in the retina.

\section{Acknowledgment}

We thank the Shanghai Biomodel Organism Science \& Technology Development Co., Ltd, which was the original source for the zebrafish.

\section{Disclosure}

The authors report no conflicts of interest in this work.

\section{References}

1. Folkman J. Tumor angiogenesis: therapeutic implications. $N$ Engl J Med. 1971;285(21):1182-1186. 
2. O'Reilly MS, Holmgren L, Shing Y, et al. Angiostatin: a novel angiogenesis inhibitor that mediates the suppression of metastases by a Lewis lung carcinoma. Cell. 1994;79(2):315-328.

3. Samant RS, Shevde LA. Recent advances in anti-angiogenic therapy of cancer. Oncotarget. 2011;2(3):122.

4. Sakurai T, Kudo M. Signaling pathways governing tumor angiogenesis. Oncology. 2011;81(Suppl 1):24-29.

5. Tampellini M, Sonetto C, Scagliotti GV. Novel anti-angiogenic therapeutic strategies in colorectal cancer. Expert Opin Investig Drugs. 2016;25(5): 507-520.

6. Manegold C. Bevacizumab for the treatment of advanced non-small-cell lung cancer. Expert Rev Anticancer Ther. 2008;8(5):689-699.

7. Sachdev JC, Jahanzeb M. Evolution of bevacizumab-based therapy in the management of breast cancer. Clin Breast Cancer. 2008; $8(5): 402-410$.

8. Fu P, He YS, Huang Q, et al. Bevacizumab treatment for newly diagnosed glioblastoma: systematic review and meta-analysis of clinical trials. Mol Clin Oncol. 2016;4(5):833-838.

9. Ferrara N, Hillan KJ, Novotny W. Bevacizumab (Avastin), a humanized anti-VEGF monoclonal antibody for cancer therapy. Biochem Biophys Res Commun. 2005;333(2):328-335.

10. Chen YX, Yang Q, Kuang JJ, et al. Efficacy of adding bevacizumab in the first-line chemotherapy of metastatic colorectal cancer: evidence from seven randomized clinical trials. Gastroenterol Res Pract. 2014;2014:594930.

11. Chavez MN, Aedo G, Fierro FA, Allende ML, Egana JT. Zebrafish as an emerging model organism to study angiogenesis in development and regeneration. Front Physiol. 2016;7:56.

12. Gore AV, Monzo K, Cha YR, Pan W, Weinstein BM. Vascular development in the zebrafish. Cold Spring Harb Perspect Med. 2012;2(5): a006684.

13. Schuermann A, Helker CS, Herzog W. Angiogenesis in zebrafish. Semin Cell Dev Biol. 2014;31:106-114.

14. Siekmann AF, Lawson ND. Notch signalling limits angiogenic cell behaviour in developing zebrafish arteries. Nature. 2007; 445(7129):781-784.

15. Covassin LD, Villefranc JA, Kacergis MC, Weinstein BM, Lawson ND. Distinct genetic interactions between multiple Vegf receptors are required for development of different blood vessel types in zebrafish. Proc Natl Acad Sci U S A. 2006;103(17):6554-6559.

16. Nicoli S, Presta M. The zebrafish/tumor xenograft angiogenesis assay. Nat Protoc. 2007;2(11):2918-2923.

17. Tan H, Mu G, Zhu W, Liu J, Wang F. Down-regulation of vascular endothelial growth factor and up-regulation of pigment epithelium derived factor make low molecular weight heparin-endostatin and polyethylene glycol-endostatin potential candidates for anti-angiogenesis drug. Biol Pharm Bull. 2011;34(4):545-550.

18. Liu L, Yu H, Huang X, et al. A novel engineered VEGF blocker with an excellent pharmacokinetic profile and robust anti-tumor activity. BMC Cancer. 2015;15:170.
19. Kimmel CB, Ballard WW, Kimmel SR, Ullmann B, Schilling TF. Stages of embryonic development of the zebrafish. Dev Dyn. 1995; 203(3):253-310.

20. Lawson ND, Weinstein BM. In vivo imaging of embryonic vascular development using transgenic zebrafish. Dev Biol. 2002;248(2):307-318.

21. Lam HW, Lin HC, Lao SC, et al. The angiogenic effects of Angelica sinensis extract on HUVEC in vitro and zebrafish in vivo. $J$ Cell Biochem. 2008;103(1):195-211.

22. Feng N, Chen H, Fu S, et al. HIF-1 $\alpha$ and HIF-2 $\alpha$ induced angiogenesis in gastrointestinal vascular malformation and reversed by thalidomide. Sci Rep. 2016;6:27280

23. Du Z, Ma HL, Zhang ZY, Zheng JW, Wang YA. Transgenic expression of a venous malformation related mutation, TIE2-R849W, significantly induces multiple malformations of zebrafish. Int J Med Sci. 2018; 15(4):385-394.

24. Tobia C, De Sena G, Presta M. Zebrafish embryo, a tool to study tumor angiogenesis. Int J Dev Biol. 2011;55(4-5):505-509.

25. Aiello LP. Angiogenic pathways in diabetic retinopathy. $N$ Engl J Med. 2005;353(8):839-841.

26. Dewan A, Liu M, Hartman S, et al. HTRA1 promoter polymorphism in wet age-related macular degeneration. Science. 2006;314(5801): 989-992.

27. Gariano RF, Gardner TW. Retinal angiogenesis in development and disease. Nature. 2005;438(7070):960-966

28. Goldsmith P, Harris WA. The zebrafish as a tool for understanding the biology of visual disorders. Semin Cell Dev Biol. 2003;14(1):11-18.

29. Carmeliet P, Jain RK. Angiogenesis in cancer and other diseases. Nature. 2000;407(6801):249-257.

30. Folkman J. Angiogenesis: an organizing principle for drug discovery? Nat Rev Drug Discov. 2007;6(4):273-286.

31. Laschke MW, Menger MD. Anti-angiogenic treatment strategies for the therapy of endometriosis. Hum Reprod Update. 2012;18(6):682-702.

32. Cook KM, Figg WD. Angiogenesis inhibitors: current strategies and future prospects. CA Cancer J Clin. 2010;60(4):222-243.

33. Hasan J, Shnyder SD, Bibby M, Double JA, Bicknel R, Jayson GC. Quantitative angiogenesis assays in vivo - a review. Angiogenesis. 2004; $7(1): 1-16$

34. Howe K, Clark MD, Torroja CF, et al. The zebrafish reference genome sequence and its relationship to the human genome. Nature. 2013; 496(7446):498-503.

35. Goel S, Duda DG, Xu L, et al. Normalization of the vasculature for treatment of cancer and other diseases. Physiol Rev. 2011;91(3): 1071-1121.

36. Serbedzija GN, Flynn E, Willett CE. Zebrafish angiogenesis: a new model for drug screening. Angiogenesis. 1999;3(4):353-359.

37. Arnold JJ. Age-related macular degeneration: anti-vascular endothelial growth factor treatment. BMJ Clin Evid. 2016;2016:pii: 0701.

38. Guo J, Glass JO, McCarville MB, et al. Assessing vascular effects of adding bevacizumab to neoadjuvant chemotherapy in osteosarcoma using DCE-MRI. Br J Cancer. 2015;113(9):1282-1288.
Drug Design, Development and Therapy

\section{Publish your work in this journal}

Drug Design, Development and Therapy is an international, peerreviewed open-access journal that spans the spectrum of drug design and development through to clinical applications. Clinical outcomes, patient safety, and programs for the development and effective, safe, and sustained use of medicines are the features of the journal, which

\section{Dovepress}

has also been accepted for indexing on PubMed Central. The manuscript management system is completely online and includes a very quick and fair peer-review system, which is all easy to use. Visit http://www.dovepress.com/testimonials.php to read real quotes from published authors. 